\title{
Goal-oriented error estimation for transient parabolic problems
}

\author{
Pedro DÍEZ ${ }^{1}{ }^{\star}$, Giovanni CALDERÓN ${ }^{2}$ \\ 1 Laboratori de Càlcul Numèric (www-lacan.upc.es), Departament de Matemàtica Aplicada III, Universitat Politècnica de \\ Catalunya, Barcelona, SPAIN. \\ 2 Departamento de Matemáticas, Facultad de Ciencias, Universidad de Los Andes, Mérida, VENEZUELA.
}

Received: date / Revised version: date

\begin{abstract}
This work focuses on controlling the error and adapting the discretization in the context of parabolic problems. In order to obtain a sound mathematical framework, the time domain is discretized using a Discontinuous Galerkin (DG) approach. This allows to formulate the time stepping procedure in a variational format. The error is measured in the basis of an output of interest of the solution, defined by a linear functional. A dual problem, associated with this linear output is introduced. The dual problem has to be solved backward in time.

An error representation is introduced, based on the weak residual of the primal error applied to the dual solution. Two different alternatives are studied to estimate the error in the dual solution: 1) recovery based error estimators and 2) implicit residual type estimators. Once the error assessment is performed implicitly in the dual problem, the obtained estimate is plugged into the primal residual to obtain the error in the quantity of interest. The implementation of the estimator is drastically simplified by using the weak version of the residual instead of the strong version used in previous works.
\end{abstract}

Thus, the output error is assessed using a mixed technique, explicit for the primal problem and implicit for the dual. In the framework of adaptive computations of transient problems, this approach is very attractive because it allows using first the implicit scheme for the dual problem and then integrating the primal problem, estimating the error explicitly and eventually adapting the space-time grid. Thus, at every time step of the time marching scheme, the estimate of the dual error is injected into the primal residual (explicit estimate for the primal problem).

\footnotetext{
* Partially supported by MCYT, Spain. Grant Contract: DPI2001-2204
}

\section{Introduction}

The modelling of transient diffusive phenomena leads to second order parabolic PDEs. This is the case of the transient heat equation, which is extensively used to simulate the thermal behavior of mechanical devices and structures. For instance, in the context of structural analysis of bridges, the loads induced by thermal effects have a significant influence in the final design. From a practical viewpoint, the thermal load is characterized by the temperature gradient in a characteristic cross section of the bridge. The temperature gradient is a simple postprocess of the temperature distribution in the cross section at a given instant $T$, say $u(\boldsymbol{x}, T)$. Thus, the quantity of interest is described by a linear functional $J(u)$.

The assessment of the error in a given quantity of interest is performed introducing a dual problem in which the linear functional $J(\cdot)$ appears in the right-hand side of the governing equation and thus plays the role of an external load. In the context of elliptic problems, the combination of standard energy error estimates for both the primal and the dual problems allows to assess the error in the output of interest $[1,2]$. In the parabolic setup, the work of Machiels [3] follows the same idea neglecting the error introduced by the time discretization. Also for parabolic problems, Rannacher and co-workers [4] explicitly express the output error in terms of residuals associated with the strong form of the primal problem and the (unknown) dual solution, following the pioneering work of Eriksson, Johnson and coworkers [5,6].

Here, the error in the quantity of interest is represented explicitly in terms of the dual solution using the weak residual. Using the weak residual instead of the strong one simplifies the implementation because there is no need of computing flux jumps across the element edges. Thus, once the error in the dual solution is estimated, it can be directly injected into the primal residual to easily obtain a sharp approximation of the error in the quantity of interest. 
The assessment of the error in the dual problem is performed using two different approaches: recovery type estimates and implicit residual type estimates. The recovery estimates are extremely simple to implement and prove to be very accurate in the application examples. The implicit residual type approach requires solving local problems in the space-time elements, involving a larger programming complexity. The latter approach is however interesting if the dual solution is non-smooth. This lack of smoothness may be caused by a number of factors. For instance, the presence of discontinuities in the data (different materials) or sharp variations in the source terms produce non-smooth (only $\mathcal{C}^{0}$, not $\mathcal{C}^{1}$ ) solutions. The residual type estimates allow capturing this kind of non-smooth error functions, while the recovery type estimates obviously fail in these situations. The performance of the two approaches is compared in the numerical examples.

The remainder of the paper is structured as follows. The model problem, the space and time discretization and the quantities of interest under consideration are presented in Sect. 2. Sect. 3 introduces the error equations and the representation of the error in the quantities of interest in terms of the primal and the dual errors. The

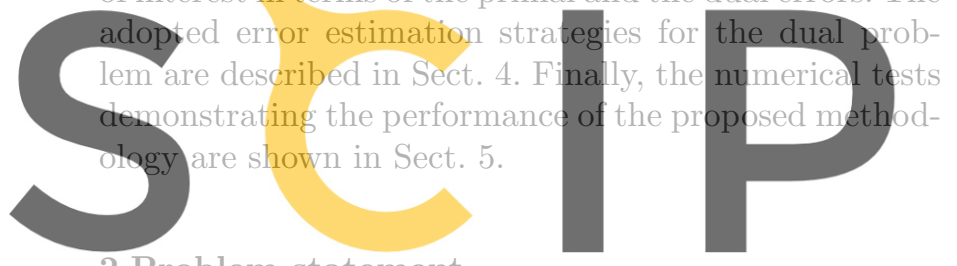

2 Problem statement

\section{Registen/forl freedeat https//www.scipedia.com to}

Let $\Omega \in \mathbb{R}^{d}$ be a bounded $d$-dimensional open domain. where $d$ is equal to 1,2 or 3 . The problem to be solved is stated as follows: find $u(\boldsymbol{x}, t)$, with $\boldsymbol{x} \in \bar{\Omega} \in \mathbb{R}^{d}$ and $t \in I:=] 0, T[$ such that

$$
\begin{aligned}
\dot{u}-\boldsymbol{\nabla} \cdot(\alpha \boldsymbol{\nabla} u) & =f & \text { in } \Omega \times I \\
u(\boldsymbol{x}, 0) & =u_{0}(\boldsymbol{x}) & \text { for } \boldsymbol{x} \in \Omega \\
u(\boldsymbol{x}, t) & =0 & \text { for } \boldsymbol{x} \in \partial \Omega, t \in I
\end{aligned}
$$

where $f$ is the source term, the coefficient $\alpha$ accounts for the thermal conductivity (the capacity and density terms affecting the $\dot{u}$ term are set to 1 ) and $\dot{u}$ stands for $\frac{\partial u}{\partial t}$. The homogeneous Dirichlet boundary condition (1c) is adopted in the presentation for the sake of simplicity. Accounting for different boundary condition types as Dirichlet non-homogeneous, Neumann or Robin does not introduce any additional conceptual difficulty. The numerical examples presented in Sect. 5 use actually different types of boundary conditions.

\subsection{Space discretization}

Problem (1) is discretized using standard Finite Elements (FE) in space. The discretization is associated with a mesh of characteristic size $H$ inducing the functional space $\mathcal{V}^{H} \subset \mathcal{H}_{0}^{1}(\Omega)$. The dependence on time is left to the coefficients (nodal unknowns) of $u^{H}$ in the FE basis:

$$
u(\boldsymbol{x}, t) \approx u^{H}(\boldsymbol{x}, t)=\sum_{i=1}^{\mathrm{n}_{\mathrm{poin}}} u_{i}(t) N_{i}(\boldsymbol{x})=\boldsymbol{u}^{\top} \boldsymbol{N}
$$

$\mathrm{n}_{\text {poin }}$ being the number of nodes in the FE mesh discretizing $\Omega, \boldsymbol{u}:=\left[u_{1}(t) u_{2}(t) \cdots u_{\mathrm{n}_{\mathrm{poin}}}(t)\right]^{\top}$ and $\boldsymbol{N}:=$ $\left[N_{1}(\boldsymbol{x}) N_{2}(\boldsymbol{x}) \cdots N_{\mathrm{n}_{\mathrm{poin}}}(\boldsymbol{x})\right]^{\top}$.

Thus, a semi-discrete form is obtained: find $u^{H}(t) \in$ $\mathcal{V}^{H}$ such that

$$
\left(\dot{u}^{H}(t), v\right)+a\left(u^{H}(t), v\right)=l(v), \text { for all } v \in \mathcal{V}^{H},
$$

where

$$
a(u, v):=\int_{\Omega} \alpha \nabla u \cdot \nabla v d \Omega, l(v):=\int_{\Omega} f v d \Omega
$$

and $(\cdot, \cdot)$ stands for the usual $\mathcal{L}_{2}$ product in $\Omega$. Equation (3) results in a system of ODEs for the time dependent coefficients (nodal unknowns) of $u^{H}$. Equation (3) is rewritten in matrix form

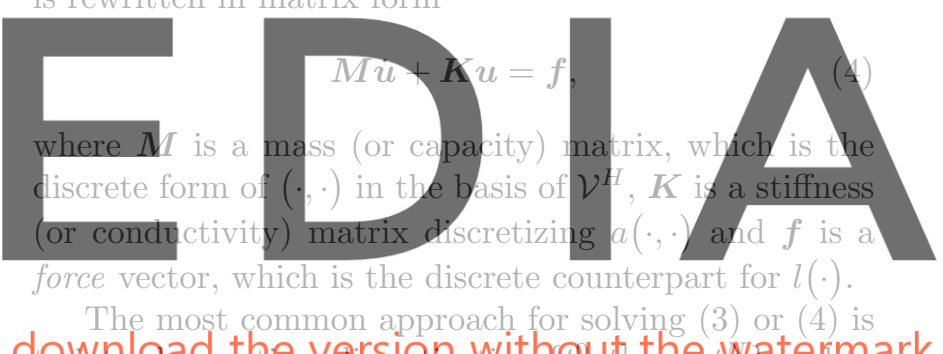

\section{domalaad thimesissiawithqut the twatermark}

use any time marching scheme yielding approximations $u^{n}$ to the nodal values vector in the corresponding times, $\boldsymbol{u}^{n} \approx \boldsymbol{u}\left(t^{n}\right)$ for $n=0,1, \ldots, N$.

In order to properly introduce the error assessment in some quantity of interest, the time discretization must be derived in a variational format. The Discontinuous Galerkin (DG) approach on time is usually adopted to fulfill this requirement $[3,4]$.

\subsection{Time discretization. Discontinuous Galerkin formulation}

As previously mentioned, a time grid is introduced: $0=$ $t^{0}<t^{1}<\cdots<t^{n}<\cdots<t^{N}=T$. The time slabs $\left.\left.I_{n}:=\right] t^{n-1}, t^{n}\right]$ and their measure $\triangle t^{n}=t^{n}-t^{n-1}$ (time steps) are also introduced for $n=1,2, \ldots, N$. Let $\triangle t$ denote the characteristic time step of the time grid. A discrete functional space on the time interval $I$ is defined associated with the time discretization:

$$
\mathcal{V}_{\triangle t}:=\left\{v: I \rightarrow \mathbb{R} ;\left.v\right|_{t \in I_{n}} \in \mathcal{P}_{q}\left(I_{n}\right)\right\},
$$

where $\mathcal{P}_{q}\left(I_{n}\right)$ stands for space of the polynomials of degree less than or equal to $q$ in $I_{n}$. Note that, in general, 
the functions in $\mathcal{V}_{\triangle t}$ are discontinuous at the points of the time grid, $t^{n}$ for $n=1,2, \ldots, N-1$.

A discrete functional space on $\Omega \times I$ is introduced combining the space and time discretizations, $\mathcal{V}^{H}$ and $\mathcal{V}_{\triangle t}$ :

$$
\begin{aligned}
\mathcal{V}_{\triangle t}^{H} & :=\{v: \Omega \times I \rightarrow \mathbb{R} \mid \\
\forall t & \left.\in I \quad v(\cdot, t) \in \mathcal{V}^{H} \text { and } \forall \boldsymbol{x} \in \Omega \quad v(\boldsymbol{x}, \cdot) \in \mathcal{V}_{\triangle t} \cdot\right\}
\end{aligned}
$$

From a practical viewpoint, taking $u^{H}$ in $\mathcal{V}_{\triangle t}^{H}$ is equivalent to choosing the coefficients $u_{i}$ in $\mathcal{V}_{\triangle t}$, see (2).

The DG solution is $u^{H} \in \mathcal{V}_{\triangle t}^{H}$ such that

$$
B\left(u^{H}, v\right)=L(v), \text { for all } v \in \mathcal{V}_{\triangle t}^{H},
$$

where

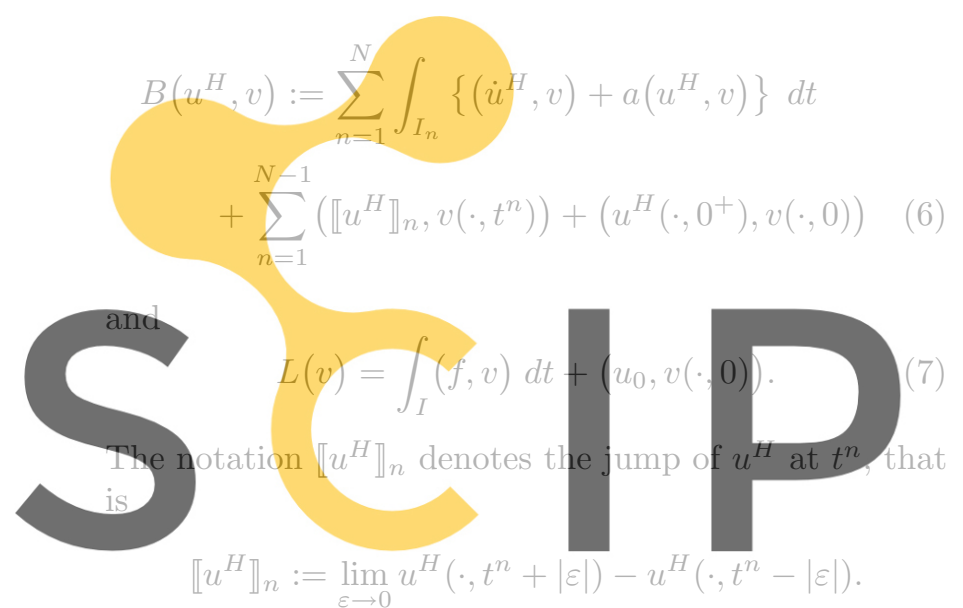

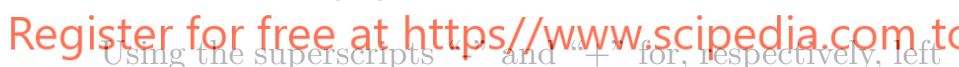
and right limits, the jump is described by

$$
\llbracket u^{H} \rrbracket_{n}:=u^{H}\left(\cdot, t^{n+}\right)-u^{H}\left(\cdot, t^{n-}\right) .
$$

The jump at $t^{0}$ is also included in (5). The value of $u^{H}$ in $t^{0-}$ is replaced by the initial condition given in (1b), $u_{0}$. The term including $u_{0}$ is in the right-hand-side of (5) because it is part of data. Once it is computed, the value of $u^{H}$ in $t^{0+}$ is different than $u_{0}$ up to the error introduced by the discretization in the numerical scheme.

Note that the broken character of $\mathcal{V}_{\triangle t}^{H}$ (discontinuous at every $t^{n}$ ) decouples the computation of $u^{H}$ in every time slab. Once $u^{H}$ is known at " $t^{(n-1)-"}$, this value is taken as an initial condition for the time slab $I_{n}$ and the following equation has to be solved:

$$
\begin{aligned}
\int_{I_{n}}\left\{\left(\dot{u}^{H}, v\right)\right. & \left.+a\left(u^{H}, v\right)\right\} d t \\
& +\left(u^{H}\left(\cdot, t^{(n-1)+}\right), v\left(\cdot, t^{n-1}\right)\right) \\
= & \int_{I_{n}}(f, v) d t+\left(u^{H}\left(\cdot, t^{(n-1)-}\right), v\left(\cdot, t^{n-1}\right)\right)
\end{aligned}
$$

for all $v$ in the restriction of $\mathcal{V}_{\triangle t}^{H}$ to the time slab $I_{n}$.

\subsection{Exact and reference solutions}

The continuous counterpart of $\mathcal{V}_{\triangle t}^{H}$ is

$$
\begin{aligned}
& \mathcal{V}:=\{v: \Omega \times I \rightarrow \mathbb{R} \mid \\
& \left.\forall t \in I \quad v(\cdot, t) \in \mathcal{H}_{0}^{1}(\Omega) \text { and } \forall \boldsymbol{x} \in \Omega \quad v(\boldsymbol{x}, \cdot) \in \mathcal{L}_{2}(I)\right\}
\end{aligned}
$$

The exact solution of (1) is also the solution of the following continuous weak equation: find $u$ such that

$$
\begin{aligned}
& \int_{I}\{(\dot{u}, v)+a(u, v)\} d t+(u(\cdot, 0), v(\cdot, 0)) \\
&=\int_{I}(f, v) d t+\left(u_{0}, v(\cdot, 0)\right),
\end{aligned}
$$

for all $v \in \mathcal{V}$. Note that $u$ is continuous along time and therefore its time jumps are zero. Consequently, equation (9) may be written as a particular case of (5). That is, $u$ is such that

$$
B(u, v)=L(v), \text { for all } v \in \mathcal{V},
$$

where the definition of $B(\cdot, \cdot)$ given in (6) is still valid because, for $u$ continuous, the jump term vanishes.

From a practical viewpoint, the error estimation strate-

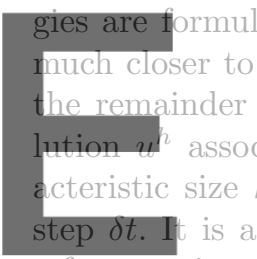

reference time grid is denoted as

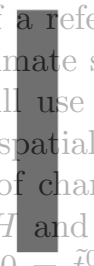
ference solution,
e solution $u{ }^{H}$.
e a refe ence so-
al mesh of char-
daracteristic time
$\tilde{t}_{0} \delta t<\Delta t$. The

$\tilde{t}^{n}<\cdots<\tilde{t}^{\tilde{N}}=T$. The time slabs $\left.\tilde{I}_{n}:=\tilde{t}^{n-1}, \tilde{t}^{n}\right]$ and

\section{downtead the version witheutothe $\mathrm{I}_{\mathrm{n}}$ watermark}

fashion. The corresponding functional space is denoted as $\mathcal{V}_{o t}^{h}$. Thus, the reference solution $u^{h} \in \mathcal{V}_{o t}^{h}$ is such that

$$
B\left(u^{h}, v\right)=L(v), \text { for all } v \in \mathcal{V}_{\delta t}^{h},
$$

where the definition of $B(\cdot, \cdot)$ must be modified

$$
\begin{aligned}
& B\left(u^{h}, v\right):=\sum_{n=1}^{\tilde{N}} \int_{\tilde{I}_{n}}\left\{\left(\dot{u}^{h}, v\right)+a\left(u^{h}, v\right)\right\} d t \\
& \quad+\sum_{n=1}^{\tilde{N}-1}\left(\llbracket u^{h} \rrbracket_{n}, v\left(\cdot, \tilde{t}^{n}\right)\right)+\left(u^{h}\left(\cdot, 0^{+}\right), v(\cdot, 0)\right)
\end{aligned}
$$

Usually, the reference time grid is taken as a refinement of the original time grid. That is, for every $n=$ $0,1,2, \ldots, N$ there exists $\tilde{n}$ such that $t^{n}=\tilde{t}^{\tilde{n}}$. In this case, the definition of $B(\cdot, \cdot)$ introduced in (6) is a particular case of the definition of (12) (they coincide for any function in $\mathcal{V}_{\triangle t}^{H}$ ).

Thus, in the following, the bilinear form $B(\cdot, \cdot)$ is taken as described in (12), that is including the jumps in all the points of the refined time grid. This definition is the most general in the sense that it unifies the writing of the equations for $u^{H}, u$ and $u^{h},(5),(10)$ and (11) respectively. 


\subsection{Quantity of interest and dual problem}

Our goal is to assess the error in some quantity of interest. We restrict ourselves to the functional outputs of the form

$$
J(u)=(j, u(\cdot, T)),
$$

that is, we are interested on a quantity depending on the solution at the final time $t=T$. The function $j(\boldsymbol{x})$ in (13) is in $\mathcal{L}_{2}(\Omega)$ and stands for the Riesz representation of the linear output.

Alternatively, it is also possible to consider an output accounting for the behavior of the solution all along the time, namely,

$$
J(u)=\int_{I} w(t)(j, u(\cdot, t)) d t
$$

where $w(t)$ is a weight function modulating the contribution of every time to the quantity of interest. For the sake of simplicity we will keep the form described in (13). However, the generalization given in (14) does not introduce any additional difficulty.

Thus, the Dual Problem is introduced as an auxiliary problem to assess the error in the quantity of interest.

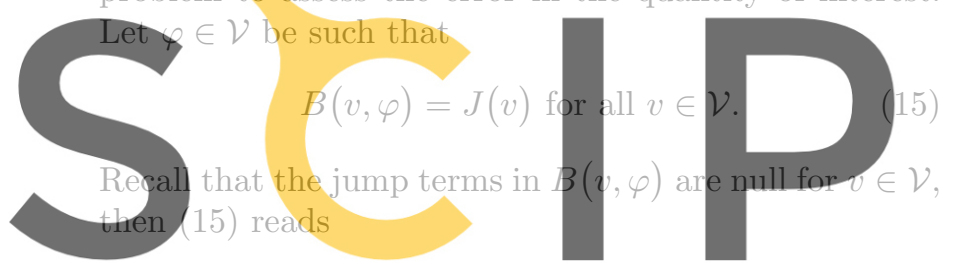

\section{Register for free at https//www.scipedia.com to} $=(j, v(\cdot, T))$ for all $v \in \mathcal{V} . \quad(16)$

After time integration by parts, (16) yields

$$
\begin{array}{r}
\int_{I}\{-(\dot{\varphi}, v)+a(\varphi, v)\} d t+(\varphi(\cdot, T), v(\cdot, T)) \\
=(j, v(\cdot, T)) \text { for all } v \in \mathcal{V} .
\end{array}
$$

Comparing (17) with (9) and (1), it is easily shown that this weak form of the dual problem is equivalent to the following strong form:

$$
\begin{aligned}
\dot{\varphi}+\boldsymbol{\nabla} \cdot(\alpha \boldsymbol{\nabla} \varphi) & =0 & \text { in } \Omega \times I \\
\varphi(\boldsymbol{x}, T) & =j(\boldsymbol{x}) & \text { for } \boldsymbol{x} \in \Omega \\
\varphi(\boldsymbol{x}, t) & =0 & \text { for } \boldsymbol{x} \in \partial \Omega, t \in I
\end{aligned}
$$

Note that the initial condition (18b) is given at $t=$ $T$. The time integration must therefore be performed backward in time. The problem is still physically and mathematically sound because the sign of the diffusion term, $\boldsymbol{\nabla} \cdot(\alpha \boldsymbol{\nabla} \varphi)$, has changed with respect to the primal problem (1).

The cost of approximating $\varphi$ is the same as approximating $u$. In the following the approximation to $\varphi$ in $\mathcal{V}^{H}, \varphi^{H}$ is considered.

\section{Error representation}

\subsection{Primal error equation}

The error $e:=u-u^{H}$ is inserted in (10) and the following weak equation for the error is derived: find $e \in \mathcal{V}$ such that

$$
B(e, v)=L(v)-B\left(u^{H}, v\right)=: R^{P}(v), \text { for all } v \in \mathcal{V},
$$

where $R^{P}(\cdot)$ stands for the weak residual in the primal problem. Using (6) and (7) the expression for $R^{P}(\cdot)$ is

$$
\begin{aligned}
R^{P}(v):=\int_{I}(f, v) d t & +\left(u_{0}, v(\cdot, 0)\right) \\
-\left(u^{H}\left(\cdot, 0^{+}\right), v(\cdot, 0)\right)-\sum_{n=1}^{N} & \int_{I_{n}}\left\{\left(\dot{u}^{H}, v\right)+a\left(u^{H}, v\right)\right\} d t \\
& -\sum_{n=1}^{N-1}\left(\llbracket u^{H} \rrbracket_{n}, v\left(\cdot, t^{n}\right)\right) \cdot
\end{aligned}
$$

Note that for any $v$ in $\mathcal{V}, R^{P}(v)$ is explicitly computed once $u^{H}$ is available. It is worth noting that the expres-

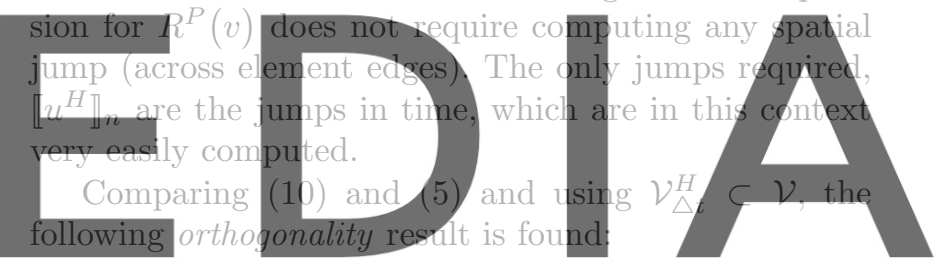

$B(e, v)=R^{P}(v)=0$, for all $v \in \nu_{\triangle t}^{H}$

(21)

download the version without the watermark

The reference error $e^{h}:=u^{h}-u^{H}$ is the solution of the discrete counterpart of (19)

$$
B\left(e^{h}, v\right)=R^{P}(v), \text { for all } v \in \mathcal{V}_{\delta t}^{h} .
$$

Moreover the orthogonality condition (21) is also verified replacing $e$ by $e^{h}\left(\right.$ recall $\left.\mathcal{V}_{\triangle t}^{H} \subset \mathcal{V}_{\delta t}^{h} \subset \mathcal{V}\right)$.

The global error equation (19) or its discrete form (22) are computationally unaffordable. The error estimation strategies based on solving these equations locally (restricted to an element or a patch of elements) yield local approximations of $e$ (or $e^{h}$ ) that can be used to evaluate energetic quantities. Generally, in order to evaluate $J(e)$, the approximation to $e$ resulting from the standard estimates is useless. However, a proper combination of energetic estimates in both the primal and the dual problems allows assessing the error in the quantity of interest, $J(e)$ or $J\left(e^{h}\right)$.

\subsection{Using $\varphi$ to assess $J(e)$}

The solution of the dual problem given by (15) and (18), $\varphi$, allows representing the error in the quantity of interest using the residual. 
Recall that $\varphi$ is in $\mathcal{V}$ and therefore $v$ can be replaced by $\varphi$ in (19), that is,

$$
B(e, \varphi)=R^{P}(\varphi)
$$

Moreover, $e$ is in $\mathcal{V}$ and therefore $v$ can be replaced by $e$ in (15), that is,

$$
B(e, \varphi)=J(e)
$$

An error representation follows from (23) and (24):

$$
J(e)=R^{P}(\varphi)
$$

This error representation gives the error in the quantity of interest, $J(e)$, as an explicit function of $\varphi$ and $u^{H}$. Recall that the residual $R^{P}(\cdot)$ depends on $u^{H}$, see (20). The residual is defined by integrals over $\Omega \times I$. Thus, using the error representation (25), the error in the quantity of interest may be easily split into local contributions, from each element and time slab.

Using the orthogonality given by (21), the r.h.s. term
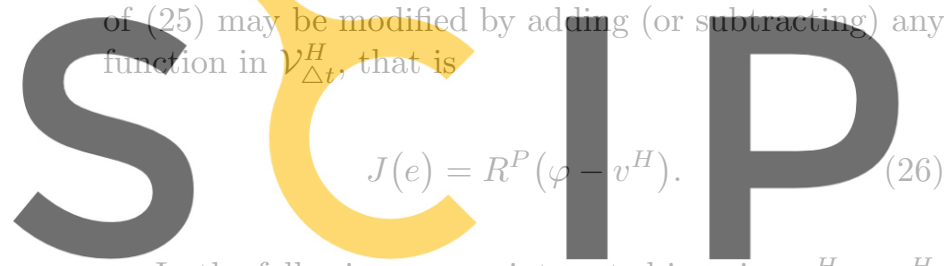

In the following we are interested in using $v^{H}=\varphi^{H}$, where $\varphi^{H}$ is the DG approximation of $\varphi$ in $\nu^{H}$, and

Register for free at https//www scipedia fom to in $\nu_{\triangle t}^{H}$. Obviously, $\varphi$ and $\Pi_{H} \varphi$ are not known but $\varphi^{H}$ can be computed in the same fashion as $u^{H}$. However, once $\varphi^{H}$ is obtained, a standard error estimate can be used to approximate $\varepsilon:=\varphi-\varphi^{H}$. The estimate for $\varepsilon$ is supposed to approximate $R^{P}(\varepsilon)$ properly because the computation of $R^{P}(\cdot)$ involves only energetic quantities.

Thus, the error representation (26) suggests using an implicit error estimation strategy for estimating the error $\varepsilon$ in the dual solution or, possibly, for $\varphi-\Pi_{H} \varphi$. Once $\varepsilon$ or $\varphi-\Pi_{H} \varphi$ are fairly approximated, the error assessment for the quantity of interest is purely explicit, see (20) and (26).

The same rationale applies if the reference solution $\varphi^{h}$, in the refined space $\mathcal{V}_{\delta t}^{h}$, is considered instead of the exact solution $\varphi$. In this case the error to be evaluated (in the quantity of interest) is $e^{h}$, represented by

$$
J\left(e^{h}\right)=R^{P}\left(\varphi^{h}-v^{H}\right), \text { for all } v^{H} \in \mathcal{V}_{\triangle t}^{H} .
$$

Then, the reference error, $J\left(e^{h}\right)$ is evaluated as a function of $\varepsilon^{h}:=\varphi^{h}-\varphi^{H}$ or $\varphi^{h}-\Pi_{H} \varphi^{h}$. The next section introduces an error estimation strategy yielding approximations for $\varepsilon^{h}$ and $\varphi^{h}-\Pi_{H} \varphi^{h}$.

\section{Error estimation}

\subsection{General strategy and requirements}

As already noted, we are concerned with approximating the solution of the dual problem. From a practical viewpoint, we focus on approximating the reference solution $\varphi^{h}$ rather than the exact solution $\varphi$. Obviously, computing $\varphi^{h}$ is equivalent to compute the reference error, $\varepsilon^{h}:=\varphi^{h}-\varphi^{H}$.

The global computation of $\varphi^{h}$ solving equation (15) in $\mathcal{V}_{\delta t}^{h}$ would result in a prohibitive computationally cost. The error estimation procedures we present in this section provides an approximation $\varphi^{\star}$ to $\varphi^{h}$, obtained after local computations and using the coarse solution $\varphi^{H}$.

In this section two different strategies are proposed to obtain $\varphi^{\star}$. First, $\varphi^{\star}$ is obtained by a simple postprocessing of $\varphi^{H}$. A recovery procedure is used following the ideas of Wiberg and coworkers [7]. A second approach is used, based on the philosophy of the residual type estimators with the simplest local boundary conditions [8]. As previously noted, this is equivalent to obtaining the corresponding error version, $\varepsilon^{\star}:=\varphi^{\star}-\varphi^{H}$.

Actually, due to the local assumptions needed to com-

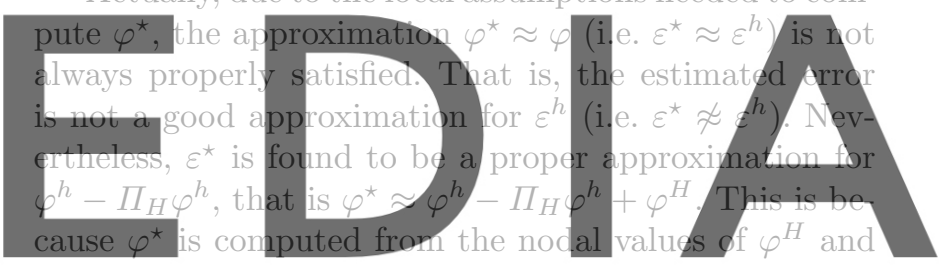

therefore the values of $\varphi^{*}$ at the nodes of the $H$-mesh dow very close tor coincide) with the values of $\varphi^{H}$ Recall

\subsection{Recovery estimates}

The first idea is to recover a function $\varphi^{\star}$ in $\mathcal{V}_{\delta t}^{h}$ from the solution $\varphi^{H}$ based on local computations. The goal is to obtain $\varphi^{\star}$ such that $\varphi^{\star} \approx \varphi^{h}$, that is to enhance the quality of $\varphi^{H}$.

Recall that $\varphi^{\star}$ is required to evaluate $R^{P}\left(\varphi^{\star}\right)$, see (20). Therefore, $\varphi^{\star}$ must be used as an argument of the $\mathcal{L}_{2}(\Omega)$ product. Thus, recovering fluxes is not sufficient. Using the mechanical language, we require displacements and not only stresses.

Previous work on recovering of displacements is due to Wiberg and coworkers [7], where the recovered solution is obtained in an enriched finite element space from the approximation in $\mathcal{V}_{\triangle t}^{H}$ by a least squares approximation.

We adopt here the same philosophy. However, some peculiarities of the present approach must be pointed out:

- We assume that the selected reference space is " $h$ refined" both in space and time. That is, there is some refinement factor $r(r=2,3,4 \ldots)$ such that 


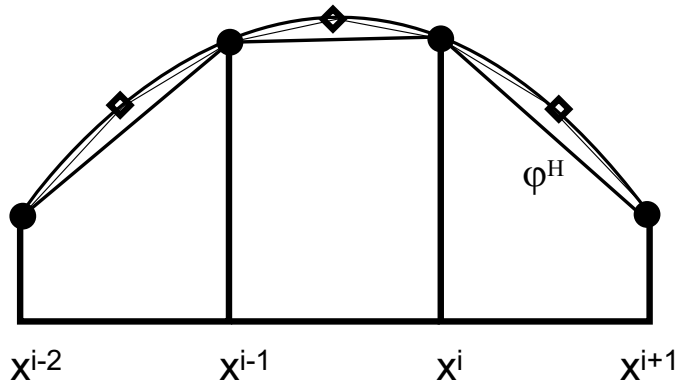

Fig. 1 Illustration of the space recovery procedure in 1D. Every element of the mesh $] x^{i-1}, x^{i}$ [ is associated with a patch $] x^{i-2}, x^{i+1}$ [ and the corresponding nodes of the $H$ mesh (marked with $\bullet$ ). A polynomial is fitted to the values in the -nodes. This polynomial is evaluated to obtain the enhanced values in the refined $h$-mesh (nodes marked with $\diamond$ )

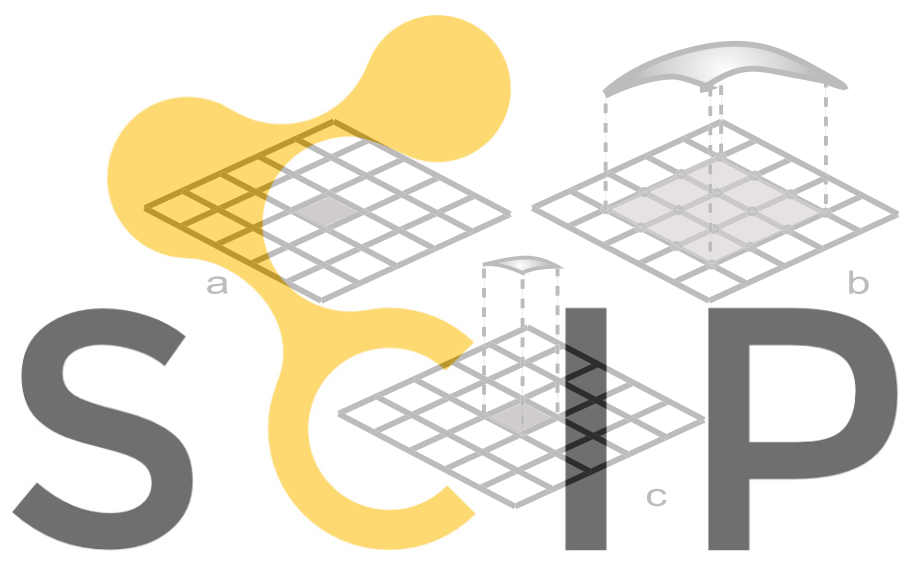

Fig. 2 Illustration of the space recovery procedure in 2D

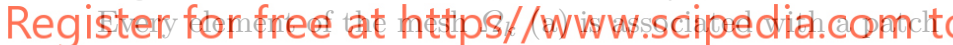
$\omega_{k}$ (shadowed in b). A polynomial is fitted to the values in the nodes in $\omega_{k}$ using a least squares criterion (b). This polynomial restricted to $\Omega_{k}$ and it is evaluated to obtain the enhanced values in the refined $h$-mesh (c).

$h=H / r$ and $\delta t=\Delta t / r$. We are not considering so far the possibility of enriching the reference space using the " $p$ " approach, that is, increasing the order of interpolation.

- The time and the space recoveries are performed independently.

- The space recovery is performed locally, in patches of elements, centered in every element of the mesh. The values of $\varphi^{H}$ at the nodes of the $H$-mesh are used as the input data and a higher order polynomial is fitted. Once the polynomial is obtained it is evaluated in the nodes of the $h$-mesh to describe $\varphi^{\star}$.

- The time recovery is also performed locally, using a patch of three time slabs. That is, to determine $\varphi^{\star}$ in $I_{n}$ we use $\varphi^{H}$ in $I_{n-1} \bigcup I_{n} \bigcup I_{n+1}$.

4.2.1 Space recovery The space recovery procedure is defined for the solution freezed at some $t \in I$. This procedure is used to recoverer an approximation $\varphi^{\star}(\cdot, t)$ in
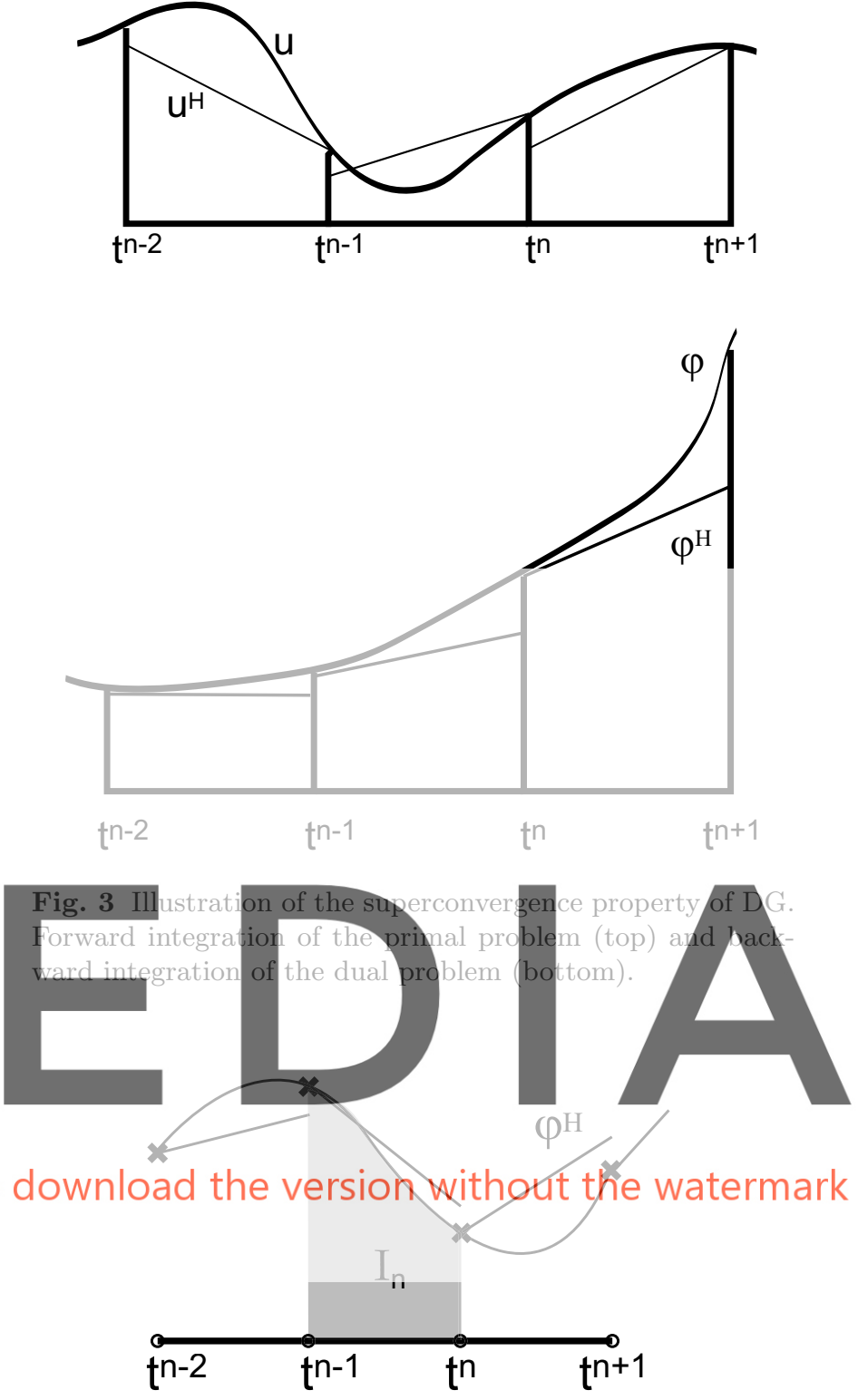

Fig. 4 Illustration of the time recovery procedure. For every time slab $I_{n}$, the previous and next time slabs are also considered to interpolate a cubic polynomial using the 4 values (in $t^{n-2}, t^{n-1}, t^{n}$ and $t^{n+1}$ ) that are assumed to be more accurate (for backward integration, the right limits).

$\mathcal{V}^{h}$ from $\varphi^{H}(\cdot, t)$. The dependence on $t$ is omitted in the following to simplify the presentation. Thus, the main idea, following [7], is to smooth out $\varphi^{H}$ locally (in some patches of elements). Every element of the mesh generating $\mathcal{V}^{H}, \Omega_{k}$ for $k=1, \ldots, \mathrm{n}_{\mathrm{elem}}$ is associated with the patch $\omega_{k}$ of elements surrounding $\Omega_{k}$, see figures 1 and 2.

In the simple 1D case represented in Fig. 1, the patch of elements involves 4 nodes of the $H$-mesh (for interior elements). In this case a cubic polynomial is interpolated using the four values of $\varphi^{H}$ in these nodes to set the values of $\varphi^{\star}$ in the nodes of the $h$-mesh. 
In the $2 \mathrm{D}$ case, a quadratic polynomial is fitted to the nodal values of $\varphi^{H}$ in $\omega_{k}$ using a least squares criterion. Following Fig. 2, the values of $\varphi^{H}$ in the 16 nodes of the $H$-mesh in $\omega_{k}$ are used to fit a quadratic polynomial (6 d.o.f. in 2D). Once this polynomial is fitted, we evaluate it in the nodes of the fine $h$-mesh in $\Omega_{k}$. The average of all the computed values is used for the nodes of the $h$ mesh involved in different patches (internal boundary of $\left.\Omega_{k}\right)$.

4.2.2 Time recovery The time recovery procedure is defined for the solution located at some $\boldsymbol{x} \in \Omega$. It consists on building up an enhanced approximation $\varphi^{\star}(\boldsymbol{x}, \cdot)$ in $\mathcal{V}_{\delta t}$ from $\varphi^{H}(\boldsymbol{x}, \cdot)$. The dependence on $\boldsymbol{x}$ is omitted in the following to simplify the presentation.

The time recovery strategy proposed here exploits a well known superconvergence property of the DG scheme. In the DG approximation the approximation in $t^{n-}$ (left limit) is much better than the approximation in $t^{n+}$. Obviously, if the time integration is performed backwards, the accurate value is $t^{n+}$. This property is standard for time DG schemes and it is illustrated in Fig. 3.

Thus, using this fact, the restriction to the time slab

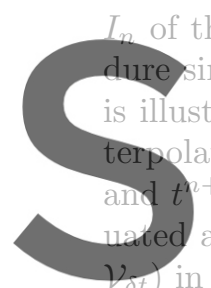
f the recovered function $\varphi$ imilar to the $1 \mathrm{D}$ spatial illustrated in Fig. 4. A cubi lating the values of $\varphi^{H}$ +1 . Once the polynomia at the points of the refined

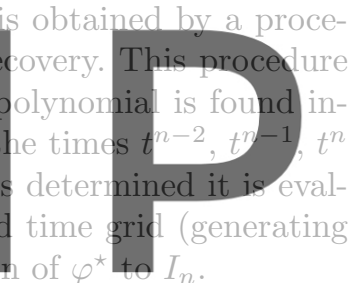

$\varphi_{0}^{\star}(\boldsymbol{x})$ from $\varphi^{H}\left(\cdot, t^{n+}\right)$ using exactly the same procedure described in Sect. 4.2.1. $\varphi_{0}^{\star}(\boldsymbol{x})$ is used as initial condition for the local problem.

- Boundary conditions on $\partial \Omega_{k} \times I_{n}$. For the sake of simplicity we set Dirichlet type boundary conditions. We also extract the boundary conditions from a recovery procedure. Now, for every node in $\partial \Omega_{k}$, we smooth out the restriction of $\varphi^{H}$ to this node, using the recovery strategy described in Sect. 4.2.2.

Once the initial and boundary conditions are set, the local problem is solved using a fine local discretization (the restriction of $\mathcal{V}_{\delta t}^{h}$ to $\Omega_{k} \times I_{n}$ ).

Note that this can be done either using $\varphi^{\star}$ or $\varepsilon^{\star}$ as unknowns. This is because the equations for $\varphi^{h}$ and $\varepsilon^{h}$ are respectively (22) and (28). Recall that both $\varphi^{\star}$ and $\varepsilon^{*}$ can be used as input of $R^{P}(\cdot)$ and the same estimate for $J(e)$ is obtained.

\subsection{Remarks on the implementation of an adaptive process}

The definition of a proper adaptive strategy is beyond the scope of this paper. However, it is worth noting that

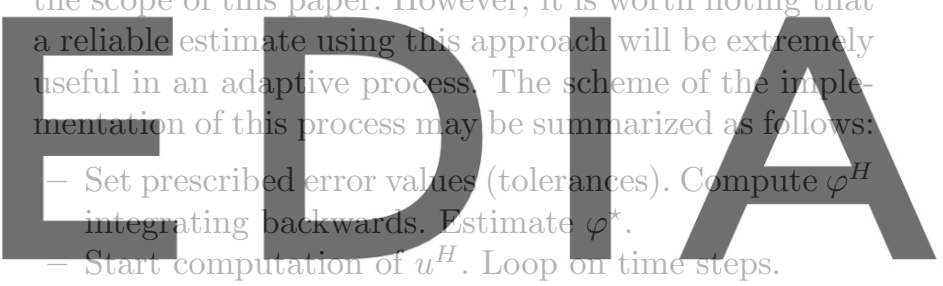

- After each time step, compute the contribution

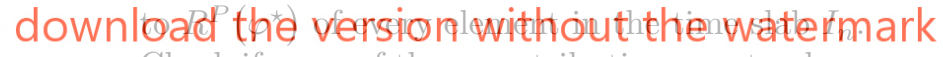

Check if some of these contributions are too large and, if needed, refine either the spatial mesh (reduce $H$ ) or the time grid (reduce $\triangle t$ ).

- End loop on time steps.

the solution of the discrete residual equation, analogous to $(22)$,

$$
B\left(v, \varepsilon^{h}\right)=R^{D}(v), \text { for all } v \in \mathcal{V}_{\delta t}^{h},
$$

where the residual in the dual problem is defined in the same fashion as $R^{P}(\cdot): R^{D}(v):=J(v)-B\left(v, \varphi^{H}\right)$. An alternative approach to build up a proper approximation to $\varepsilon^{h}$ is to solve locally the residual equation (28).

The main difficulty for this kind of estimates is to set proper boundary conditions to the local problems.

Here, we present a simple approach based on solving the restriction of (28) to every element of the space-time mesh, that is to every $\Omega_{k} \times I_{n}$ for $k=1,2, \ldots, \mathrm{n}_{\mathrm{elem}}$ and $n=1,2, \ldots, N$.

In this case we need both initial conditions at $\Omega_{k} \times$ $\left\{t^{n}\right\}$ (recall that for $\varphi$ the time integration is performed backwards) and boundary conditions on $\partial \Omega_{k} \times I_{n}$. Here we have made the following choices:

- Initial condition at $\Omega_{k} \times\left\{t^{n}\right\}$. We assume, as in 4.2.2, that at $t=t^{n}, \varphi^{H}\left(\cdot, t^{n+}\right)$ is much more accurate than $\varphi^{H}\left(\cdot, t^{n-}\right)$. Thus we recover an approximation

\section{Numerical examples}

5.1 Example 1: 1D synthetic problem

\begin{tabular}{|c|c|c|c|c|}
\hline$J(u)$ & $J\left(u^{h}\right)$ & $J\left(u^{H}\right)$ & $J(e)$ & $J\left(e^{h}\right)$ \\
\hline 0.3369 & 0.3367 & 0.3361 & $8.09 \times 10^{-4}$ & $6.09 \times 10^{-4}$ \\
\hline
\end{tabular}

Table 1 Example 1: Values of the quantity of interest for the solutions and errors

We consider the $1 \mathrm{D}$ spatial domain $\Omega:=] 0,1[$ and time interval $I=] 0,0.2[$, i.e. $T=0.2$. We solve Problem (1) with $\alpha=1$ and $f$ such that the exact solution is

$$
u(x, t):=\mathrm{e}^{-\pi^{2}\left(t+t^{2}\right)} \sin (\pi x),
$$

see Fig. 5. The solution $u$ is such that (1b) is verified and the initial condition is set such that the solution 


\begin{tabular}{|c|c|c|c|}
\hline & $R^{P}(\cdot)$ & $R^{P}(\cdot) / J(e)$ & $R^{P}(\cdot) / J\left(e^{h}\right)$ \\
\hline$\varphi_{1}^{\star}$ (recov.) & $6.09 \times 10^{-4}$ & $75.3 \%$ & $100.1 \%$ \\
$\varphi_{2}^{\star}$ (resid.) & $6.07 \times 10^{-4}$ & $75.0 \%$ & $99.8 \%$ \\
$\varphi^{h}$ & $6.09 \times 10^{-4}$ & $75.2 \%$ & $100.0 \%$ \\
$\varphi$ & $8.09 \times 10^{-4}$ & $100.0 \%$ & $110.4 \%$ \\
\hline
\end{tabular}

Table 2 Example 1: Residuals giving approximations to errors in the quantity of interest. Reference solution with $r=2$. Effectivity indices.

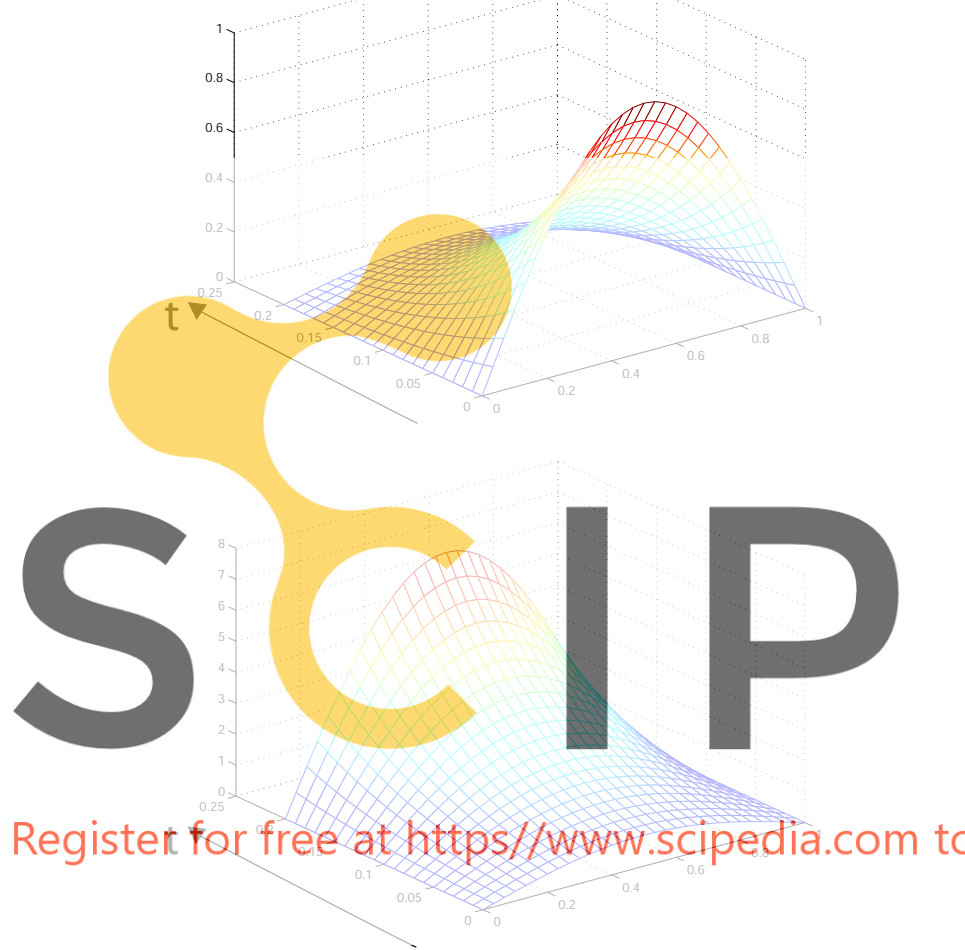

Fig. 5 Example 1: Representation of the primal solution $u^{H}$ (top) and the dual solution $\varphi^{H}$ (bottom))

holds, that is $u_{0}(x)=u(x, 0)$. The quantity of interest is defined according to (13) taking

$$
j(x)=\mathrm{e}^{\pi^{2} T} \sin (\pi x),
$$

namely, the quantity of interest is a weighted average of the solution at the last time, at $t=T$. The approximations $u^{H}$ and $\varphi^{H}$ are computed with a uniform mesh of 30 two-noded linear elements. The time grid is uniform with $N=20$ and therefore $\Delta t=T / N=0.01$. The solutions $u^{H}$ and $\varphi^{H}$ are displayed in Fig. 5.

The reference mesh is both determined by a refining factor of $2(r=2)$, see Tab. 2 , and $6(r=6)$, see Tab. 3 . Recall that $h=H / r$ and $\delta t=\Delta t / r$.

For each value of $r$, two different recovered solutions, $\varphi_{1}^{\star}$ and $\varphi_{2}^{\star}$, are obtained, both in $\mathcal{V}_{\delta t}^{h}$. The first one is computed following the strategy described in Sect. 4.2 (recovery) and the latter following the strategy introduced in Sect. 4.3 (residual).

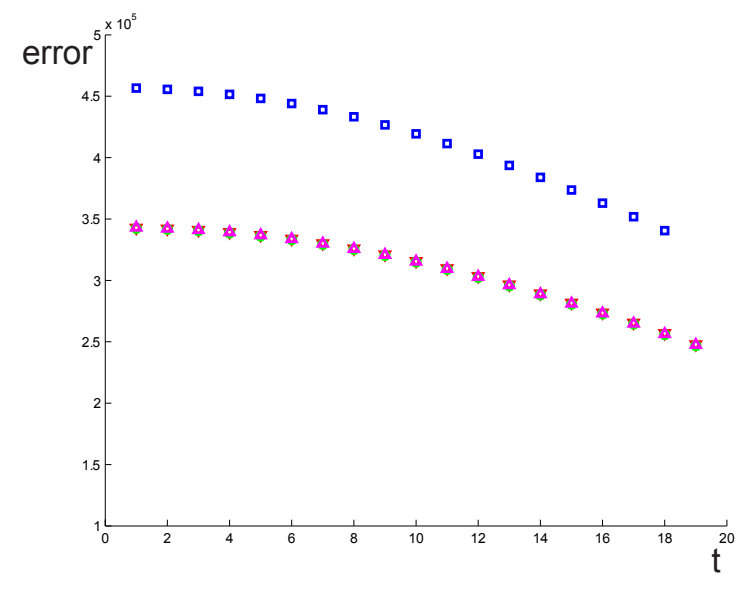

Fig. 6 Example 1: Error distribution along time. Contributions to the error from every time slab corresponding to exact error $\square$, reference error $\Delta$, estimated error with the recovery approach $\nabla$ and estimated error with the residual approach $*$. Note that the latter three (reference error and two estimates) are practically identical

The numerical results are summarized in tables 1,2 and 3. It can be noted, comparing tables 1 and 2 , that

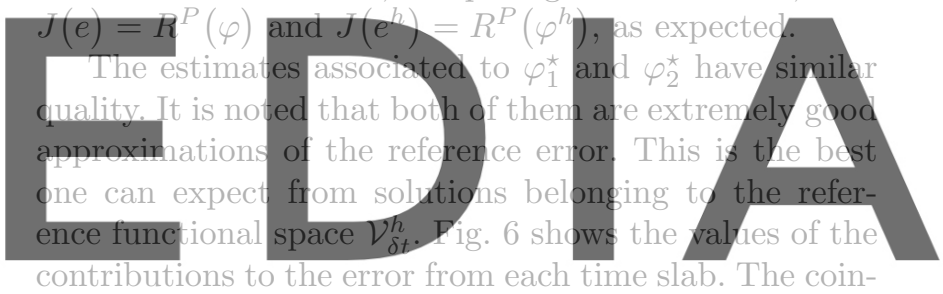

cidences between $\varphi_{1}^{*}$ and $\varphi_{2}^{*}$ and the reference error are

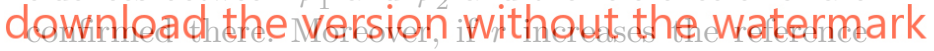
solution gets closer to the exact solution. The estimates follow the same behavior as shown in Tab. 3 .

\begin{tabular}{|c|c|c|c|}
\hline & $R^{P}(\cdot)$ & $R^{P}(\cdot) / J(e)$ & $R^{P}(\cdot) / J\left(e^{h}\right)$ \\
\hline$\varphi_{1}^{\star}$ (recov.) & $7.86 \times 10^{-4}$ & $97.1 \%$ & $99.7 \%$ \\
$\varphi_{2}^{\star}$ (resid.) & $7.87 \times 10^{-4}$ & $97.3 \%$ & $100.0 \%$ \\
$\varphi^{h}$ & $7.87 \times 10^{-4}$ & $97.3 \%$ & $100.0 \%$ \\
$\varphi$ & $8.09 \times 10^{-4}$ & $100.0 \%$ & $102.8 \%$ \\
\hline
\end{tabular}

Table 3 Example 1: Residuals giving approximations to errors in the quantity of interest. Reference solution with $r=6$. Effectivity indices.

\subsection{Example 2: 2D synthetic problem}

We consider the 2D spatial domain $\Omega:=] 0,1[\times[0,1]$ and time interval $I=] 0,0.2[$, i.e. $T=0.2$. We solve Problem (1) with $\alpha=1$ and $f$ such that the exact solution is

$u(x, y, t):=100 \mathrm{e}^{-10 t-800\left(x-x_{m}\right)^{2}\left(y-y_{m}\right)^{2}} \sin (\pi x) \sin (\pi y)^{2}$, where $x_{m}=y_{m}=0.1$. The solution $u$ is such that (1b) is verified and the initial condition is set such that the 

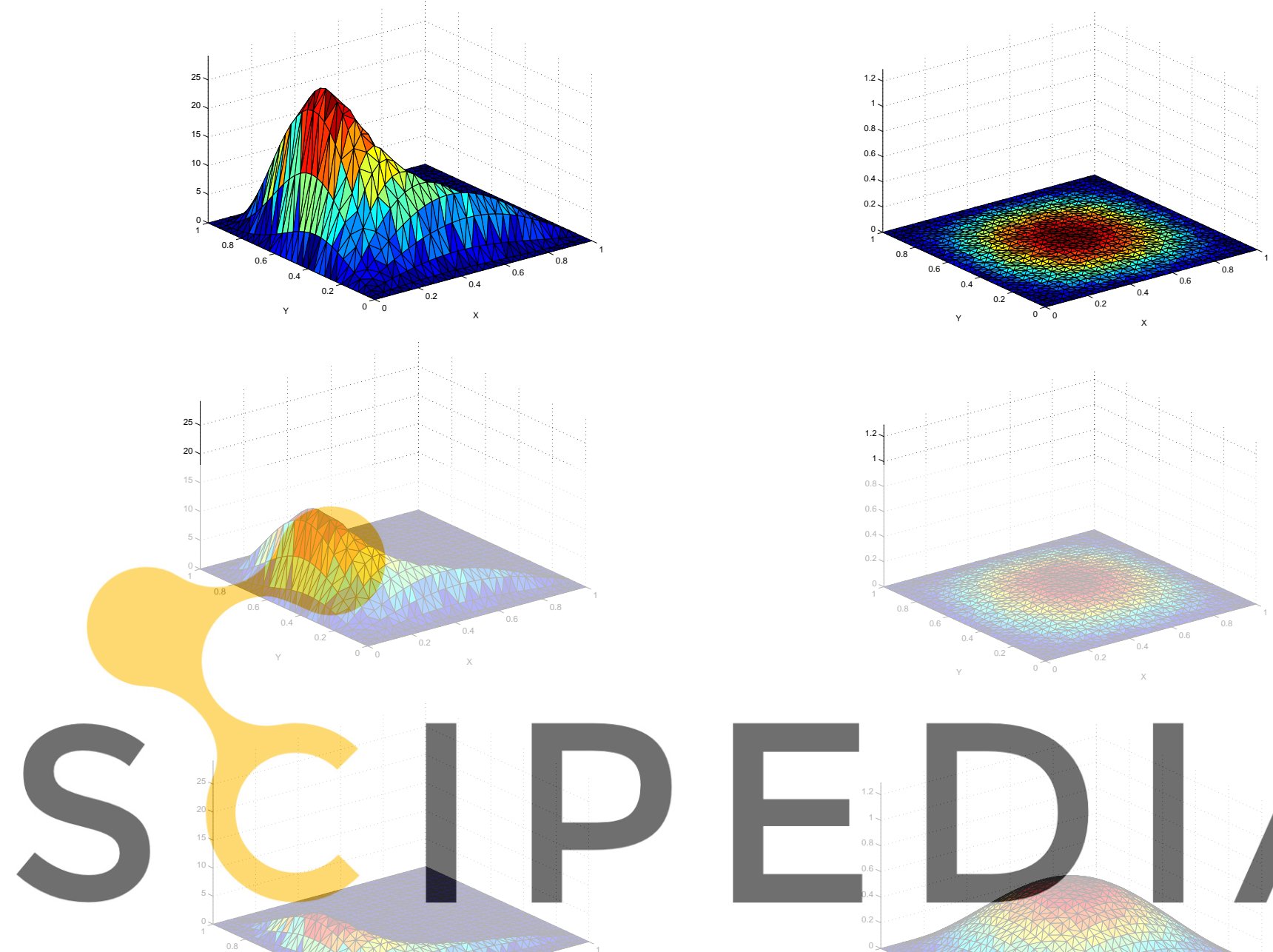

Register for free at https//www.s sipedia.com to download the version without the watermark

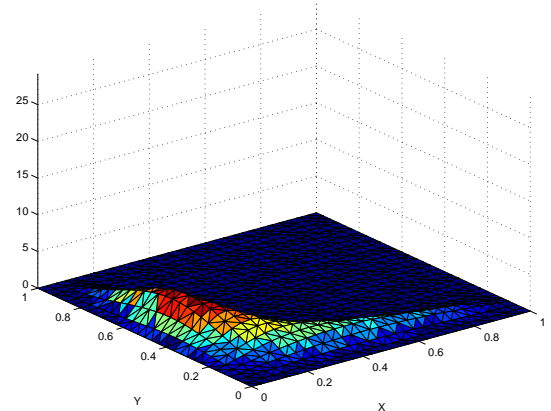

Fig. 7 Example 2: Representation of the solution $u^{H}$ at different times $(t=2 \triangle t, t=8 \triangle t, t=16 \triangle t$ and $t=20 \triangle t=T$, from top to bottom)
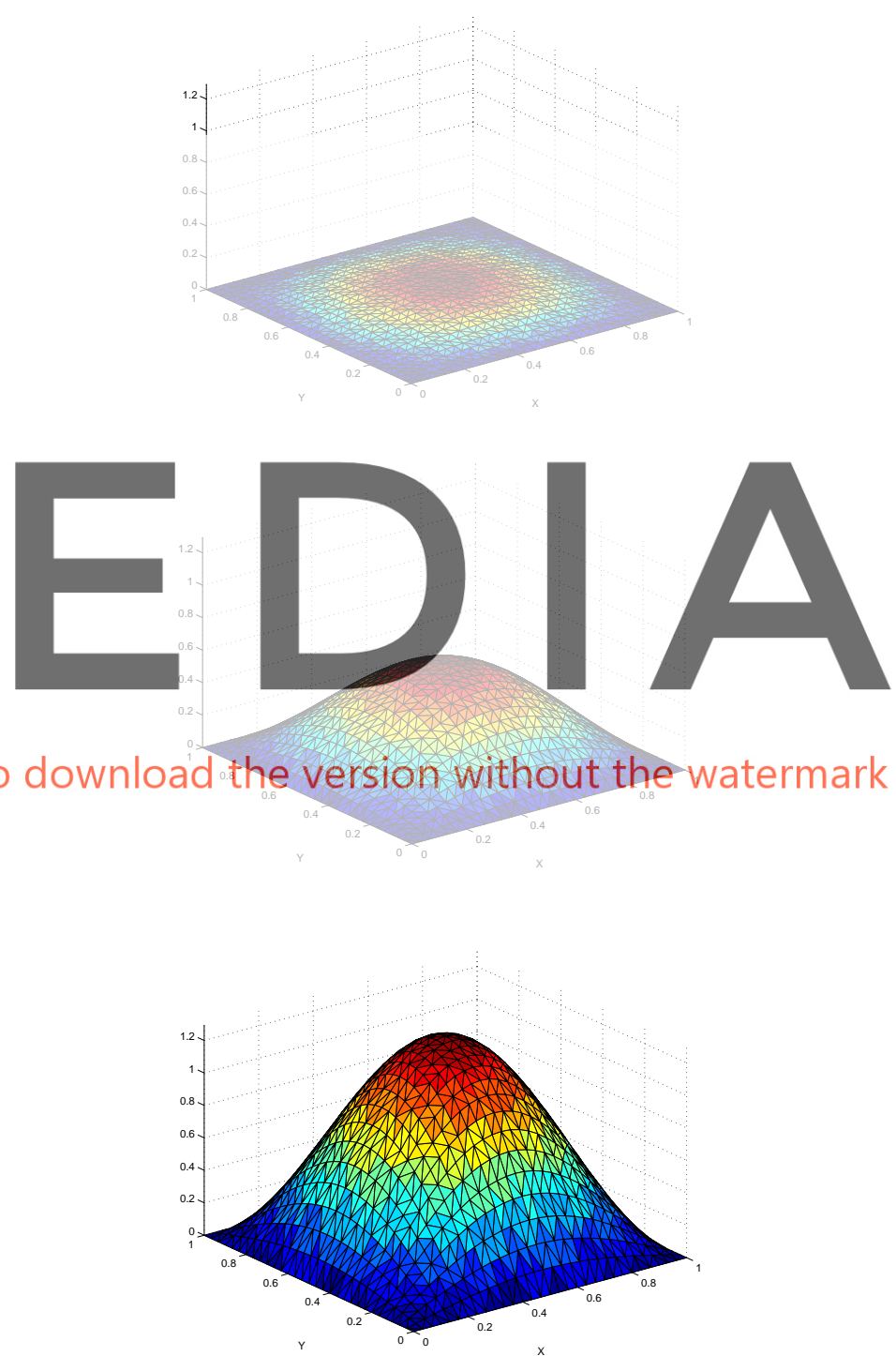

Fig. 8 Example 2: Representation of the solution $\varphi^{H}$ at different times $(t=2 \Delta t, t=8 \Delta t, t=16 \triangle t$ and $t=20 \triangle t=T$, from top to bottom) 


\begin{tabular}{|c|c|c|c|c|}
\hline$J(u)$ & $J\left(u^{h}\right)$ & $J\left(u^{H}\right)$ & $J(e)$ & $J\left(e^{h}\right)$ \\
\hline 0.2601 & 0.2601 & 0.2597 & $0.463 \times 10^{-3}$ & $0.356 \times 10^{-3}$ \\
\hline
\end{tabular}

Table 4 Example 2: Values of the quantity of interest for the solutions and errors

\begin{tabular}{|c|c|c|c|}
\hline & $R^{P}(\cdot)$ & $R^{P}(\cdot) / J(e)$ & $R^{P}(\cdot) / J\left(e^{h}\right)$ \\
\hline$\varphi_{1}^{\star}$ (recov.) & $3.46 \times 10^{-4}$ & $74.8 \%$ & $97.2 \%$ \\
$\varphi_{2}^{\star}$ (resid.) & $4.26 \times 10^{-4}$ & $92.0 \%$ & $119.6 \%$ \\
$\varphi^{h}$ & $3.56 \times 10^{-4}$ & $76.9 \%$ & $100.0 \%$ \\
$\varphi$ & $4.60 \times 10^{-4}$ & $100.0 \%$ & $129.4 \%$ \\
\hline
\end{tabular}

Table 5 Example 2: Estimated errors associated with different approximations of the dual solution $\varphi$ and effectivity indices with respect to the exact and the reference solutions.

solution holds, $u_{0}(x, y)=u(x, y, 0)$. The quantity of interest is defined according to (13) taking

$$
j(x, y)=\frac{1}{40} \exp \left(2 \pi^{2} T\right) \sin (\pi x) \sin (\pi y) .
$$

The approximations $u^{H}$ and $\varphi^{H}$ are computed with a uniform mesh of $30 \times 30$ four-noded quadrilateral elements. The time grid is uniform with $N=20$ and there-

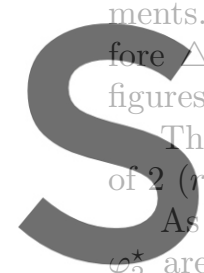
$=T / N=0.01$ The

gures 7 and 8.

he reference mesh is deter

$=2)$. Thus, $h=H / 2$ an

in the previous examp
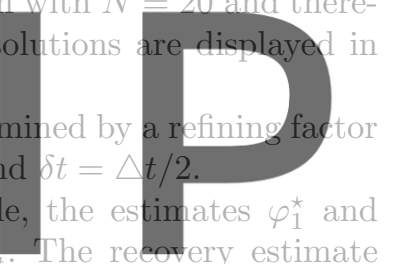

$\varphi_{1}^{\star}$ follows the strategy described in Sect. 4.2 and the

Registedufopsfreed a

The numerical results are summarized in tables 4 and 5. It can be noted, also in this example, comparing tables 4 and 5 , that $J\left(e^{h}\right)=R^{P}\left(\varphi^{h}\right)$ and $J(e)=R^{P}(\varphi)$, as expected. Some examples show a tiny difference between $J(e)$ and $R^{P}(\varphi)$. This is due to the error introduced by the numerical quadrature in the integration of the exact solution, which is not a polynomial. Here, this error is below the threshold of accuracy used for displaying the results.

The behavior of the recovery estimate is, in this example, similar to the previous one. The effectivity corresponding to the reference error is very good (97.2\%). The residual estimate is not so sharp, presenting an effectivity index of approximately $92.0 \%$ w.r.t. the exact solution and $119.6 \%$ w.r.t. the reference solution.

Fig. 9 shows the spatial error distribution at a given time (at different times the results are similar). The exact, reference and estimated (both with the recovery and the residual approaches) error distributions are practically identical, both in shape and values. The space distributions depicted in Fig. 9 are the local restrictions (to $\Omega_{k}$ and the corresponding time slab) of the residual $R^{P}(\cdot)$ evaluated in $\varphi, \varphi^{h}, \varphi_{1}^{\star}$ and $\varphi_{2}^{\star}$, respectively for the exact error, the reference error, the recovery estimate and the residual estimate.
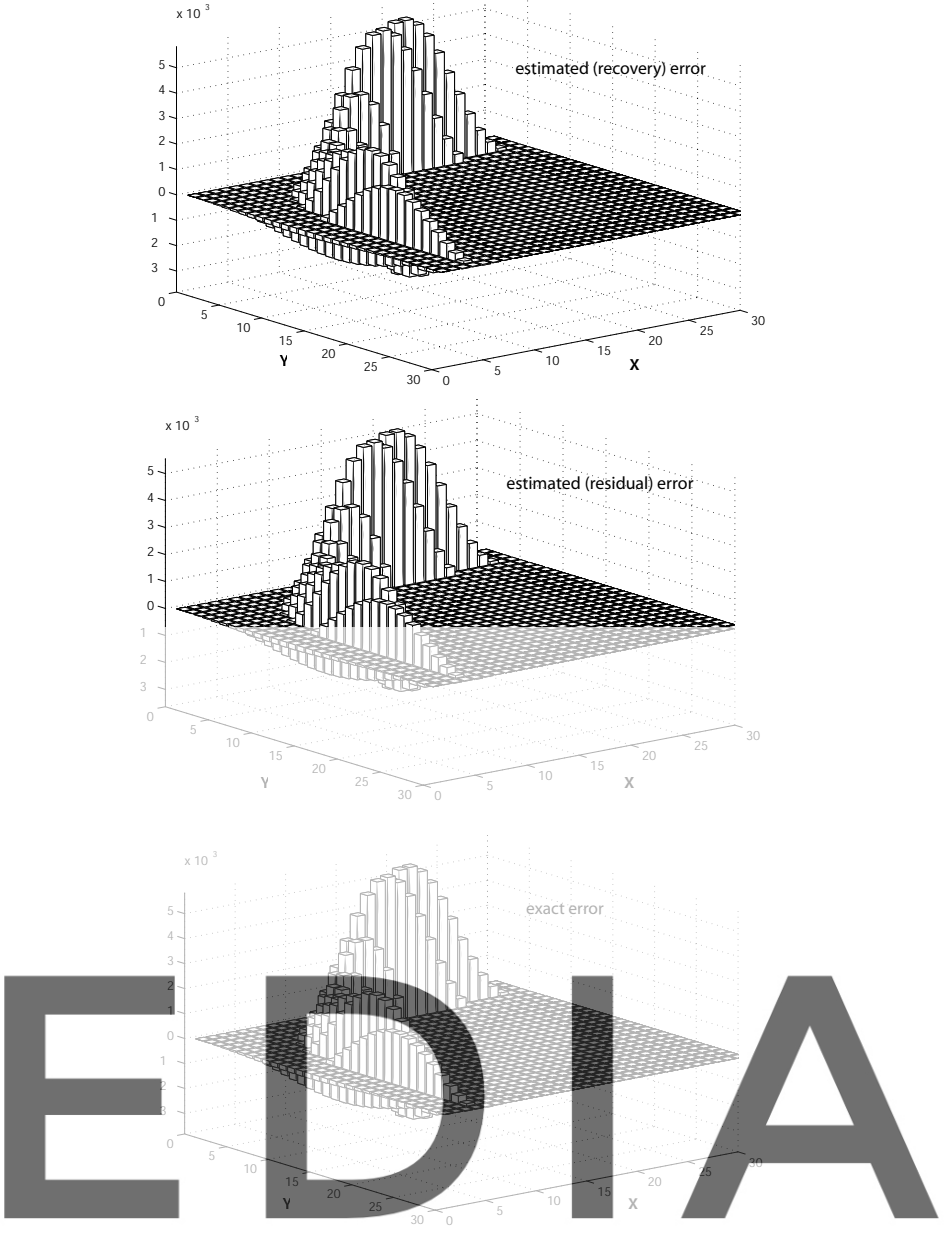

\section{download the version without the watermark}

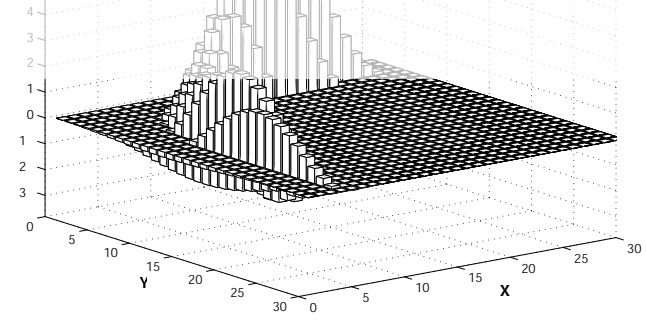

Fig. 9 Example 2: Error distribution at $t=16 \triangle t$. Distributions estimated by recovery approach (top), estimated by residual approach, exact error and reference error (bottom). All are practically identical

In this example, the recovery estimate performs extremely well, both for the value of the quantity of interest and the local contributions to the error. Moreover, recall that the recovery estimate is computationally less costly than the residual estimate.

These results motivate the use of the recovery estimate in the following example. 


\subsection{Example 3: thermal effects on a bridge cross-section}

The evolution of the temperature distribution in a bridge cross-section is analyzed. The geometry of the crosssection is displayed in Fig. 10. The thermal parameters are set to the following values corresponding to concrete:

- thermal conductivity $\kappa=1.5 \mathrm{~W} / \mathrm{m}{ }^{\circ} \mathrm{C}$

- density $\rho=2500 \mathrm{~kg} / \mathrm{m}^{3}$

- specific heat $c=960 \mathrm{~J} / \mathrm{kg}^{\circ} \mathrm{C}$

This implies solving the model problem (1) with $\alpha=$ $\kappa /(\rho c)=6.25 \times 10^{-7} \mathrm{~m}^{2} / \mathrm{s}$ and $f=0$ (no heat production in the concrete body). In this example, the Dirichlet boundary condition (1c) is now replaced by a Robin type boundary condition

$$
(\kappa \nabla u) \cdot n=I+h_{c}\left(u_{A}-u\right)
$$

where $I$ stands for the heat flux induced by the solar radiation (it is of course a function of time and it acts only in the top part of the boundary, $\left.\Gamma_{1}\right), h_{c}$ is a coefficient representing the convection (it is taken uniform all along the boundary and equal to $15 \mathrm{~J} / \mathrm{m} \mathrm{s}^{\circ} \mathrm{C}$ ) and $u_{A}$ is the external temperature (varying with time).

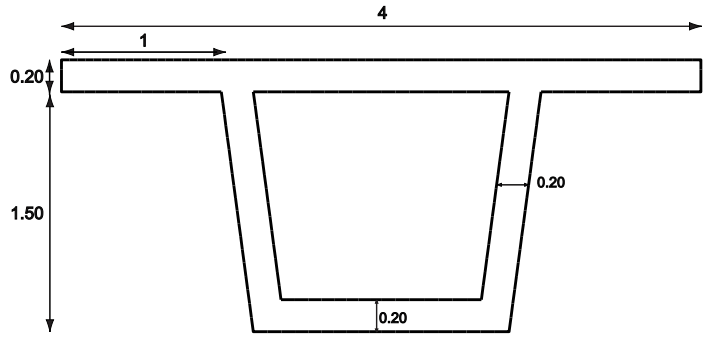

Fig. 10 Example 3: geometry of the bridge cross-section, lengths expressed in meters

The evolution of $I$ and $u_{A}$ as functions of time is described by

$$
\begin{array}{r}
I(t)= \begin{cases}\frac{2 W}{T_{0}} \sin ^{2}\left(\frac{\pi}{T_{0}}\left(t-t_{a}\right)\right) & \text { for } t_{a} \leq t \leq t_{a}+T_{0}, \\
0 & \text { otherwise }\end{cases} \\
\text { and } \quad u_{A}(t)=u_{M}+\frac{\Delta_{U}}{2} \sin \left(\frac{\pi}{12}\left(t-t_{m}\right)\right),
\end{array}
$$

where $T_{0}$ is the duration of the day light (it is set to 10 hours), $t_{a}$ is the time of dawn (it is set to 8:00 am) and $W$ accounts for the total amount of energy given by the insolation per length unit (it is set to $7000 \mathrm{~J} / \mathrm{m}$ ), $u_{M}$ is the average ambient temperature, $\Delta_{U}$ is the temperature range (difference maximum minus minimum temperatures) and 12 stands for the duration of a half day (in these expressions time is expressed in hours). Note that the natural time unit is the hour and consequently, the magnitudes involving time (e.g. $\alpha$ ) must be properly expressed in terms of hours, that is $\alpha=6.25 \times 10^{-7} \mathrm{~m}^{2} / \mathrm{s}$ $=2.25 \times 10^{-3} \mathrm{~m}^{2} /$ hour .

The mechanical load induced by the thermal effects is characterized by the so-called temperature gradient, which is the slope of a plane fitting the actual temperature distribution, see Fig. 11. This temperature gradient is considered as the quantity of interest, that has to be assessed accurately. Formally, the linear function $u_{L}(x, y)$ equivalent to the temperature distribution $u(x, y)$ (at a given time $t$ ) is obtained using a least squares fitting. Once $u_{L}(x, y)=a_{0}+a_{x} x+a_{y} y$ is determined, the quantity of interest is precisely the coefficient $a_{y}$.

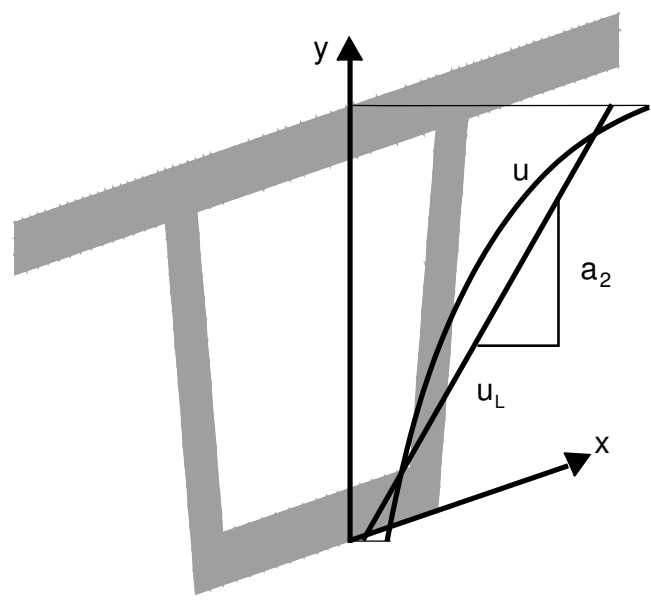

Fig. 11 Example 3: illustration of the quantity of interest.

An orthogonal basis of the linear polynomials in the domain $\Omega$ is obtained in order to easily compute $a_{y}$ as a direct function of $u$. Let $P_{0}(x, y)=1$ and $P_{1}(x, y)$ and $P_{2}(x, y)$ be obtained using a Gram-Schmidt orthogonalization from the family $\{1, x, y\}$, that is

$$
P_{1}(x, y)=x-\frac{\int_{\Omega} x P_{0} d \Omega}{\int_{\Omega} P_{0} P_{0} d \Omega} P_{0}=x-\frac{\int_{\Omega} x d \Omega}{\text { meas } \Omega}=x-\bar{x}
$$

and

$$
P_{2}(x, y)=y-\underbrace{\frac{\int_{\Omega} y d \Omega}{\operatorname{meas} \Omega}}_{\bar{y}}-\frac{\int_{\Omega} y(x-\bar{x}) d \Omega}{\int_{\Omega}(x-\bar{x})^{2} d \Omega}(x-\bar{x}),
$$

where $(\bar{x}, \bar{y})$ is the center of gravity of the cross-section. Thus, $u_{L}$ is explicitly computed as

$u_{L}=\frac{\int_{\Omega} u P_{0} d \Omega}{\int_{\Omega} P_{0} P_{0} d \Omega} P_{0}+\frac{\int_{\Omega} u P_{1} d \Omega}{\int_{\Omega} P_{1} P_{1} d \Omega} P_{1}+\frac{\int_{\Omega} u P_{2} d \Omega}{\int_{\Omega} P_{2} P_{2} d \Omega} P_{2}$

and, $a_{y}$ coincides with the coefficient affecting $P_{2}$, that is

$$
a_{y}=\frac{\int_{\Omega} u P_{2} d \Omega}{\int_{\Omega} P_{2} P_{2} d \Omega}
$$


Note that the quantity of interest is $J(u)=a_{y}$ for a given time $t=T$ (we will adopt $T$ as the end of the computation). Thus, the definition of the functional output describing the quantity of interest is precisely

$J(u)=\int_{\Omega} u(\cdot, T) j d \Omega \quad$ where $\quad j=\frac{P_{2}}{\int_{\Omega} P_{2} P_{2} d \Omega}$,

which is exactly the form given in Eq. (13).

If the geometry is symmetric with respect to the $y$ axis, the expression is further simplified since

$$
\bar{x}=0, \quad P_{1}(x, y)=x \quad \text { and } \quad P_{2}(x, y)=y-\bar{y} .
$$

In this case

$$
j(x, y)=\frac{y-\bar{y}}{\int_{\Omega}(y-\bar{y})^{2} d \Omega} .
$$

The computation starts at midnight, when the temperature is assumed to be uniform and it is carried out for 63 hours ( $T=63$ hours). That is, the last time is 3:00 pm of the third day. From a practical viewpoint, after two day cycles the solution is assumed to be independent of the initial conditions and therefore not polluted by the errors introduced by setting $u_{0}$. At 3:00 pm the quantity of interest is assumed to reach its maximum (and therefore critical) value. The time evolution of the temperature gradient is described in Fig. 12 both for the solution $u^{H}$ and the reference solution $u^{h}$. It is worth noting that the maximum is indeed reached daily around 3:00 pm Fig. 13 shows the distribution of temperature at the end of the computation. The output of interest is precisely the gradient associated with this distribution. Note that the high temperatures are concentrated at the top of the cross-section, due to the effect of solar radiation.

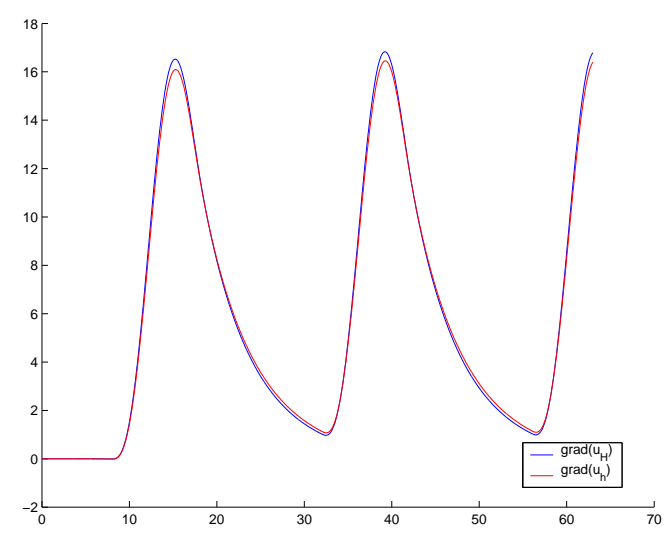

Fig. 12 Example 3: evolution of the temperature gradient both for $u^{H}$ and $u^{h}$

Here the error is estimated using the recovery estimate introduced in Sect. 4.2. The error assessment is performed for the solution obtained with the two meshes

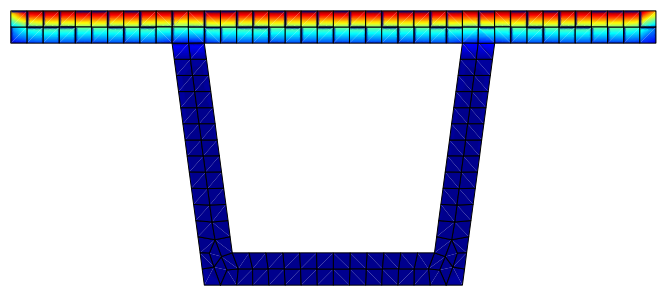

Fig. 13 Example 3: temperature distribution at time $t=T$ (end of computation)

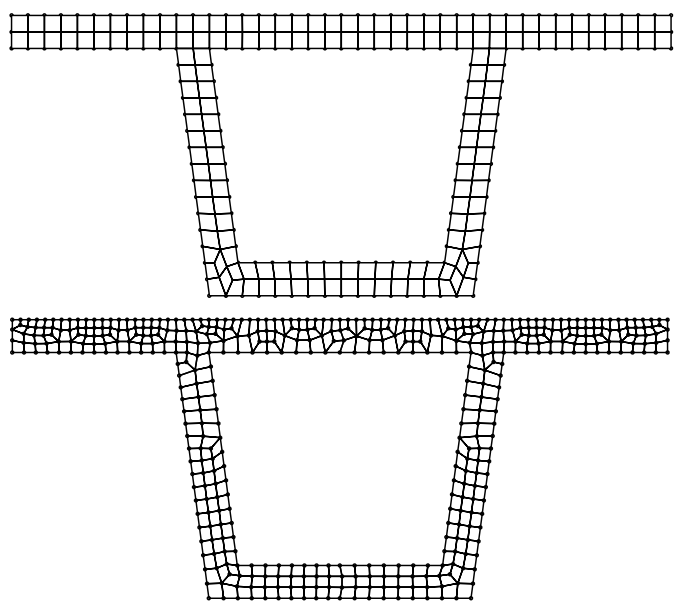

Fig. 14 Example 3: Meshes used in the computation, with 168 elements (top) and 368 elements (bottom)

displayed in Fig.14 ( of 168 elements and 368 elements) and with the following values for $\triangle t$ (in hours): $\triangle t=0.02$; $0.1 ; 0.2 ; 0.7 ; 1 ; 1.5$ and 3 (corresponding to 3150,630 , $315,90,63,42$ and 21 time steps, respectively). Moreover, the error assessment is performed for two reference meshes, with half element size and time step $(r=2)$ and with $r=4$. The estimated errors are computed in all cases, however the computational cost of obtaining the reference solution is prohibitive for $r=4$ and $\Delta t=0.02$ and also with the mesh of 368 elements for $r=4$.

\begin{tabular}{|c|cc|}
\hline$\triangle t$ & $\begin{array}{c}\text { Mesh 1 } \\
\text { 168 elements }\end{array}$ & $\begin{array}{c}\text { Mesh 2 } \\
368 \text { elements }\end{array}$ \\
\hline 0.02 & 16.79108 & 16.48149 \\
0.1 & 16.79108 & 16.48149 \\
0.2 & 16.79113 & 16.48153 \\
0.7 & 16.79268 & 16.48292 \\
1 & 16.79544 & 16.48539 \\
1.5 & 16.80692 & 16.49591 \\
3 & 16.85808 & 16.53685 \\
\hline
\end{tabular}

Table 6 Example 3: Values of $J\left(u^{H}\right)$ for all computations

The results are summarized in the following tables. Tab. 6 shows the values of $J\left(u^{H}\right)$ in all the test cases. Tab. 7 displays the reference error in all cases where it could be computed, that is, the values of $-J\left(e^{h}\right) / J\left(u^{h}\right)$ 


\begin{tabular}{|c|cc|cc|}
\hline \multirow{2}{*}{$\triangle t$} & \multicolumn{2}{|c|}{ Mesh 1 } & \multicolumn{2}{c|}{ Mesh 2 } \\
& \multicolumn{2}{|c|}{168 elements } & \multicolumn{2}{c|}{368 elements } \\
& $r=2$ & $r=4$ & $r=2$ & $r=4$ \\
\hline 0.02 & $2.402 \%$ & - & $0.934 \%$ & - \\
0.1 & $2.402 \%$ & $2.954 \%$ & $0.934 \%$ & - \\
0.2 & $2.402 \%$ & $2.954 \%$ & $0.935 \%$ & - \\
0.7 & $2.410 \%$ & $2.964 \%$ & $0.942 \%$ & - \\
1 & $2.425 \%$ & $2.980 \%$ & $0.955 \%$ & - \\
1.5 & $2.490 \%$ & $3.050 \%$ & $1.015 \%$ & - \\
3 & $2.722 \%$ & $3.357 \%$ & $1.190 \%$ & - \\
\hline
\end{tabular}

Table 7 Example 3: Values of the reference relative error, $-J\left(e^{h}\right) / J\left(u^{h}\right),-$ if not available

\begin{tabular}{|c|cc|cc|}
\hline \multirow{3}{*}{$\Delta t$} & \multicolumn{2}{|c|}{ Mesh 1 } & \multicolumn{2}{c|}{ Mesh 2 } \\
& \multicolumn{2}{|c|}{168 elements } & \multicolumn{2}{c|}{368 elements } \\
& $r=2$ & $r=4$ & $r=2$ & $r=4$ \\
\hline 0.02 & 0.42161 & 0.52636 & 0.16813 & 0.21016 \\
0.1 & 0.42162 & 0.52638 & 0.16814 & 0.21016 \\
0.2 & 0.42172 & 0.52649 & 0.16821 & 0.21024 \\
0.7 & 0.42501 & 0.53021 & 0.17112 & 0.21350 \\
1 & 0.42941 & 0.53506 & 0.17477 & 0.21746 \\
1.5 & 0.44187 & 0.54772 & 0.18563 & 0.22821 \\
3 & 0.51574 & 0.51574 & 0.17062 & 0.21208 \\
\hline
\end{tabular}

Table 8 Example 3 : Values of the estimated error, $-R^{P}\left(\varphi^{\star}\right)$

\begin{tabular}{|c|cc|cc|}
\hline \multirow{2}{*}{$\triangle t$} & \multicolumn{2}{|c|}{ Mesh 1 } & \multicolumn{2}{c|}{ Mesh 2 } \\
& \multicolumn{2}{|c|}{168 elements } & \multicolumn{2}{c|}{368 elements } \\
& $r=2$ & $r=4$ & $r=2$ & $r=4$ \\
\hline 0.02 & $107.1 \%$ & - & $110.2 \%$ & - \\
0.1 & $107.1 \%$ & $109.3 \%$ & $110.2 \%$ & - \\
0.2 & $107.1 \%$ & $109.3 \%$ & $110.2 \%$ & - \\
0.7 & $107.5 \%$ & $109.7 \%$ & $111.2 \%$ & - \\
1 & $108.0 \%$ & $110.1 \%$ & $112.1 \%$ & - \\
1.5 & $108.2 \%$ & $110.1 \%$ & $112.0 \%$ & - \\
3 & $94.2 \%$ & $94.2 \%$ & $87.8 \%$ & - \\
\hline
\end{tabular}

Table 9 Example 3: Values of the effectivity index w.r.t the reference error, $R^{P}\left(\varphi^{\star}\right) / J\left(e^{h}\right)$, - if not available

(we introduce a minus sign because all values are originally negative). Tab. 8 shows the estimated error in the quantity of interest, $R^{P}\left(\varphi^{\star}\right)$, both for $r=2$ and $r=4$. Finally, the effectivity indices computed for all cases where the reference error is computationally affordable are shown in Tab. 9.

It is worth noting from Tab. 6 that the error introduced by the time discretization is negligible for $\Delta t=$ 0.1 and $\triangle t=0.02$. This is confirmed in Tab. 7, moreover for mesh 1 (168 elements), the time error associated with $\triangle t=0.2$ is also negligible with regard to the error introduced by the mesh.

The results in Tab. 7 indicate also that the reference error is proportional to the exact error by a factor (the ratio exact/reference error) that depends on the refining index, $r$. For large $r$, the reference error is practically equal to the reference error. For the values of $r$ that re- sult in a non-expensive local description of the error, the ratio exact/reference depends on the convergence rates and may be fairly approximated using the principle of Richardson's extrapolation, see [8].

The estimated errors are displayed in Tab. 8. The behavior of the estimated error quantities is consistent, for a given mesh the error increases with $\triangle t$. The only anomaly is detected for mesh 2 (368 elements) and $\triangle t=$ 3 , where the error is reduced from $\Delta t=1.5$. This is due to the fact that the dependence of the quantity of interest with time is far from being monotonic. Thus, the large time steps (3 hours!) may yield errors with different signs.

Tab. 9 shows the effectivity indices w.r.t. the reference solution. Obviously, these values are available only in the cases where the reference solution is computed. The values of the effectivity index range from 88 to $112 \%$, that is the difference between the exact error and the estimated error is lower than $12 \%$ in all cases.
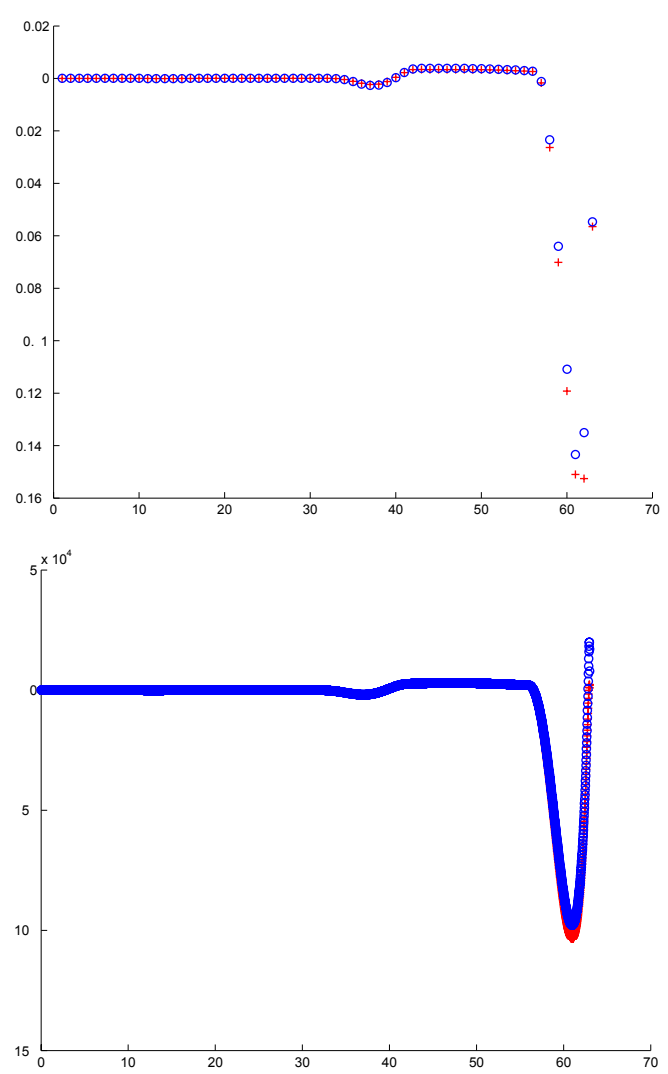

Fig. 15 Example 3: Time-distribution of the contributions to the error in the quantity of interest. Contribution from every time slab to the error. Both the reference values (o) and the estimated values $(+)$ are represented for two cases: Mesh 1 (168 elements), $\triangle t=1$ and $r=4$ (top) and Mesh 2 (368 elements), $\triangle t=0.02$ and $r=2$ (bottom).

This good behavior stands also locally, both in space and time, as shown in Fig. 15 and Fig. 16 The distribution of the contributions to the error in every time 

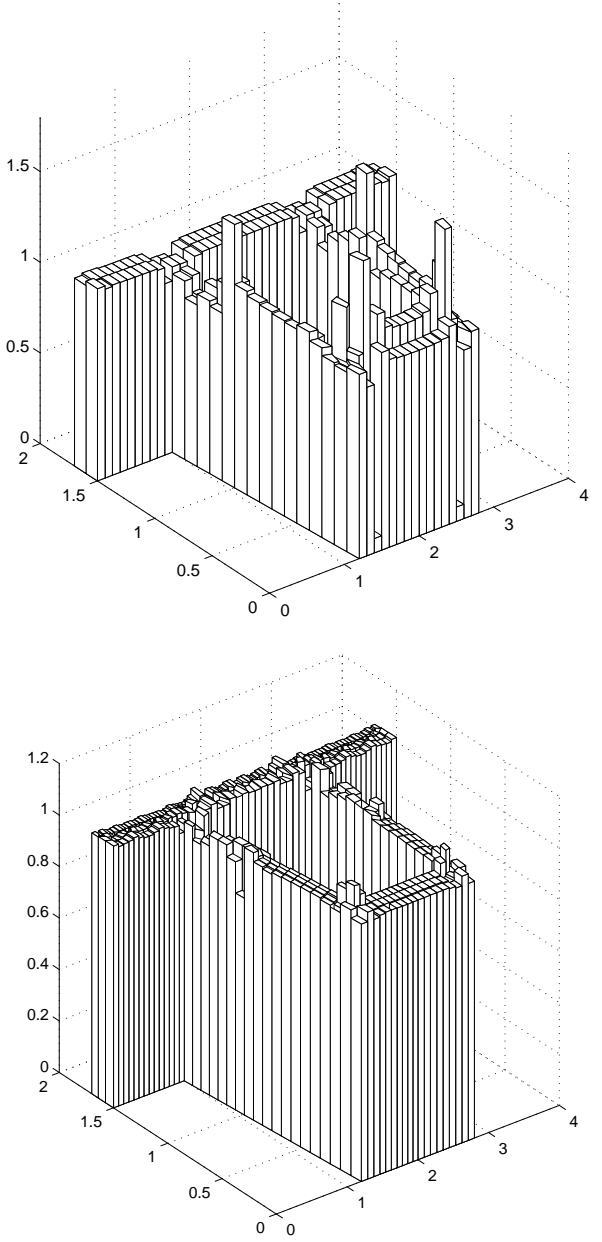

Fig. 16 Example 3: Local effectivity indices of the spacedistribution of the contributions to the error in the quantity of interest. Contribution from every element to the error, accumulated in time. Two cases: Mesh 1 (168 elements), $\triangle t=1$ and $r=4$ (top) and Mesh 2 (368 elements), $\triangle t=0.02$ and $r=2$ (bottom).

slab (accumulated in space) is assessed very accurately. In Fig. 15 the results demonstrate that the estimated values of the time contributions are very good approximations to the reference errors. Only two of the test cases are displayed, the behavior being similar for the other studied cases.

The space distribution is the restriction to every element $\Omega_{k}$ of the residual, see Eq. (26) and Eq. (26). That is, the contribution of every element of the mesh to the error in the quantity of interest, accumulated in time. This spatial representation of the error is obviously not unique: the local representation of the r.h.s term of Eq. (26) depends on the selected $v^{H}$. Different options for $v^{H}$ lead to different error distributions. The choice of the optimal $v^{H}$ in order to properly drive an adaptive procedure is beyond the scope of this paper. Thus, Fig. 16 shows the distribution of the local effectivity index associated with such spatial quantities, that is the estimated value divided by the reference values. The local effectivity indices are all close to one and the distribution is quite uniform. Fig. 16 demonstrates that the proposed error estimator produces also sharp estimates for the spatial error distributions, which is a key aspect in an adaptive framework. The accuracy of the spatial error assessment is also similar for all other test cases.

\section{Concluding remarks}

The paper introduces a straightforward and efficient methodology to estimate the error in the quantities of interest for second order parabolic problems. The main idea is to produce a recovery estimate for the dual solutions and to plug it into the residual of the primal problem. This approach is especially interesting in view of an adaptive procedure.

An alternative residual estimate is also considered, which increases the computational cost and the complexity of the implementation. In the studied examples the residual estimator does not show any advantage, the results being of the same level of accuracy. However, the residual estimator is expected to be much more efficient than the recovery estimator in problems with singular features (material discontinuities, sharp loads...) where the solution is not regular.

The results in the analyzed examples, both academical and practical, demonstrate the performance of the proposed approach.

\section{References}

1. Marius Paraschivoiu, Jaime Peraire, and Anthony T. Patera. A posteriori finite element bounds for linearfunctional outputs of elliptic partial differential equations. Comput. Methods Appl. Mech. Engrg., 150(1-4):289-312, 1997. Symposium on Advances in Computational Mechanics, Vol. 2 (Austin, TX, 1997).

2. J. T. Oden and S. Prudhomme. Goal-oriented error estimation and adaptivity for the finite element method. Comput. Math. Appl., 41(5-6):735-756, 2001.

3. L. Machiels. A posteriori finite element bounds for output functionals of discontinuous galerkin discretizations of parabolic problems. Computer Methods in Applied Mechanics and Engineering, 190:3401-3411, 2001.

4. W. Bangerth and R. Rannacher. Adaptive Finite Element Methods for Differential Equations. Birkhäuser Verlag AG, 2003.

5. K. Eriksson and C. Johnson. Adaptive finite element methods for parabolic problems I: A linear model problem. SIAM J. Numer. Anal., 28:43-77, 1991.

6. K. Eriksson, C. Johnson, and A. Logg. Encyclopedia of Computational Mechanics, volume 1, chapter 24 (Adaptive Computational Methods for Parabolic Problems). John Wiley \& Sons, 2004.

7. N.-E. Wiberg, L.F. Zeng, and X.D. Li. Error estimation and adaptivity in elastodynamics. Computer Methods in Applied Mechanics and Engineering, 101:369-395, 1992. 
8. P. Díez, J.J. Egozcue, and A. Huerta. A posteriori error estimation for standard finite element analysis. Computer Methods in Applied Mechanics and Engineering, 163:141$157,1998$. 